\title{
Complicações clínicas e obstétricas em pacientes com eclampsia com e sem recorrência de convulsões após tratamento com sulfato de magnésio
}

\section{Clinical and obstetric complications in patients with eclampsia with and without recurrence of convulsions after magnesium sulfate treatment}

Fernanda de Macêdo Silva ${ }^{1}$. Raimundo Homero de Carvalho Neto ${ }^{2}$. Francisco Edson de Lucena Feitosa ${ }^{3}$.

1 Médica residente do Programa de Ginecologia e Obstetrícia, Universidade Federal do Ceará (UFC), Fortaleza, Ceará, Brasil. 2 Médico Ginecologista e Obstetra, Maternidade Escola Assis Chateaubriand (MEAC), Fortaleza, Ceará, Brasil. 3 Doutorado em Tocoginecologia, Médico Ginecologista e Obstetra, Professor Adjunto do Departamento de Saúde Materno-Infantil, Universidade Federal do Ceará (UFC), Fortaleza, Ceará, Brasil.

\section{RESUMO}

Objetivo: determinar a prevalência de complicações clínicas e obstétricas em pacientes eclâmpticas com e sem recorrência de crises convulsivas após administração do sulfato de magnésio. Metodologia: através de um coorte retrospectivo, foram avaliados 69 casos de eclampsia atendidos na Maternidade Escola Assis Chateaubriand entre julho/2013 e julho/2016. Os testes $\mathrm{x}^{2}$ de associação e exato de Fisher foram utilizados para a comparação de variáveis. Resultados: seis pacientes apresentaram recorrência da eclampsia após sulfato de magnésio na admissão $(8,7 \%)$, sendo administrado diazepam em uma, fenitoína em quatro e metade da dose de ataque do sulfato na última. Não houve diferença significativa, entre os grupos com ou sem a presença de recorrência, na idade ( $\mathrm{p} 0,655)$, paridade ( $\mathrm{p} 0,07)$ e hipertensão crônica associada ( $\mathrm{p} 0,758)$. Também não houve diferença significativa entre os dois grupos em relação à síndrome HELLP (hemólise, elevação de enzimas hepáticas e plaquetopenia) (33,3\% x 20,6\%,p 0,389), insuficiência respiratória $(16,7 \%$ x $6,3 \%$, p 0,832$)$ e descolamento prematuro da placenta $(0 \%$ x 6,3\%, p 0,689). Conclusão: a recorrência de crise convulsiva $(8,7 \%)$ foi similar à relatada na literatura mundial $(9,7 \%)$. Não houve aumento de complicações clínicas e obstétricas nas pacientes com recorrência de eclampsia.

Palavras-chave: Sulfato de magnésio. Eclampsia. Recorrência. Gravidez de alto risco.

\section{ABSTRACT}

Objective: To determine the prevalence of clinical and obstetric complications in eclamptic patients with and without recurrence of seizures after magnesium sulfate administration. Methodology: Through a retrospective cohort, 69 cases of eclampsia attended at Maternidade Escola Assis Chateaubriand between July/2013 and July/2016 were evaluated. Fisher's exact association and $\mathrm{x}^{2}$ tests were used to compare variables. Results: Six patients presented recurrence of eclampsia after magnesium sulfate on admission (8.7\%), diazepam was administrated in one, phenytoin in four and half of the sulfate dose in the last one. There was no significant difference between groups with or without recurrence in age ( $\mathrm{p} 0.655)$, parity ( $\mathrm{p} 0.07)$ and associated chronic hypertension ( $\mathrm{p}$ 0.758). There was also no significant difference between the two groups in relation to HELLP syndrome (hemolysis, elevated liver enzyme levels, and low platelet levels) (33.3\% vs. $20.6 \%$, p 0.389), respiratory failure (16.7\% vs. $6.3 \%$, p 0.832$)$ and placental abruption $(0 \%$ vs. $6.3 \%$, p 0.689$)$. Conclusion: The recurrence of seizures $(8.7 \%)$ was similar to that reported in the world literature $(9.7 \%)$. There was no increase in clinical and obstetric complications in patients with recurrent eclampsia.

Keywords: Magnesium sulfate. Eclampsia. Recurrence. High-risk pregnancy.

Autor correspondente: Fernanda de Macêdo Silva, Avenida Senador Virgílio Távora, 77, Meireles, Fortaleza, Ceará. CEP: 60170-265. Telefone: +55 88 99950-4210. E-mail: fernanda.m.silva@gmail.com

Conflito de interesses: Não há qualquer conflito de interesses por parte de qualquer um dos autores.

Recebido em: 06 Out 2017; Revisado em: 11 Dez 2017; Aceito em: 10 Jan 2018. 


\section{INTRODUÇÃO}

As desordens hipertensivas da gestação, incluindo pré-eclâmpsia, complicam por volta de $10 \%$ das gestações, representando uma das maiores causas de morbidade e mortalidade materna e fetal ao redor do mundo. ${ }^{1}$

A incidência de pré-eclâmpsia tem aumentado cerca de $25 \%$ nos Estados Unidos, nas duas últimas décadas e as pacientes acometidas podem evoluir, em casos mais graves, para quadro de eclampsia, edema agudo de pulmão, síndrome HELLP (hemólise, elevação de enzimas hepáticas e plaquetopenia), acidente vascular cerebral e oligúria. ${ }^{2}$

A eclampsia se manifesta pela presença de um ou mais episódios de crise convulsiva tônico-clônica generalizada e/ou coma, em gestante com pré-eclâmpsia, na ausência de doenças neurológicas. A causa das convulsões ainda não está bem definida. Entre as teorias propostas estão o vasoespasmo cerebral com isquemia local, a encefalopatia hipertensiva com hiperperfusão, o edema vasogênico e a lesão endotelial. ${ }^{3}$ Nos países desenvolvidos, a eclampsia ocorre mais raramente, enquanto que nos países em desenvolvimento sua frequência é maior, parecendo estar relacionada ao subdiagnóstico e falha no tratamento da pré-eclâmpsia. No Brasil, estima-se uma incidência em torno de um caso para cada 1.000 partos. $^{4}$

A eclampsia é uma intercorrência emergencial que demanda monitorização intensiva. Os cuidados iniciais requerem suporte respiratório e controle dos episódios de convulsão. O parto é indicado logo após a estabilização clínica da paciente, uma vez que a má adaptação placentária é a principal responsável pela fisiopatologia do quadro.,

Além da resolução da gestação, é recomendada a utilização do sulfato de magnésio. Esta é a droga de escolha tanto na profilaxia, como no tratamento das convulsões eclâmpticas. ${ }^{7}$ O uso do sulfato de magnésio para o tratamento da eclampsia foi primeiramente utilizado por Lazard, em 1925. Posteriormente também foi recomendado para a prevenção da instalação de crises convulsivas em gestantes com pré-eclâmpsia grave. ${ }^{8}$

Mesmo os ensaios clínicos multicêntricos e as revisões sistemáticas assegurando a efetividade e a segurança do sulfato de magnésio na redução significativa dos episódios convulsivos, observa-se recorrência das convulsões em $10-20 \%$ dos casos. Segundo a literatura, existe aumento significativo da morbimortalidade materna quando há persistência dos episódios de convulsão, surgindo complicações como síndrome HELLP, hemorragia cerebral, falência cardiopulmonar, insuficiência renal aguda, acidose e sepse. ${ }^{9}$ Sendo assim, o objetivo deste estudo foi determinar a prevalência de complicações clínicas e obstétricas depois da recorrência de crises convulsivas após administração do sulfato de magnésio, comparando-a com o grupo sem recorrência. Pretendemos também descrever o tratamento utilizado na recorrência das crises convulsivas.

\section{PACIENTES E MÉTODOS}

O estudo foi realizado através da análise retrospectiva de prontuários de pacientes admitidas na Maternidade Escola Assis Chateaubriand (MEAC) com diagnóstico de eclampsia no período de julho/2013 a julho/2016.

A MEAC é um serviço de referência em atenção terciária em obstetrícia no estado do Ceará. Neste hospital é priorizado o atendimento e suporte adequado à gestante de risco elevado. A pré-eclâmpsia, como a principal causa de morte materna no Brasil, está no perfil de pacientes atendidas na MEAC, sendo as mesmas encaminhadas dos hospitais de baixa complexidade de Fortaleza e do interior do estado do Ceará.

Para o atual estudo, foi considerada paciente eclâmptica aquela com convulsões tônico-clônicas generalizadas, com aumento da pressão arterial após a $20^{\mathrm{a}}$ semana gestacional, sem história prévia de convulsão fora da gestação. A hipertensão foi caracterizada como pressão arterial sistólica (PAS) $\geq 140$ $\mathrm{mmHg}$ e/ou pressão arterial diastólica $(\mathrm{PAD}) \geq 90 \mathrm{mmHg}$. Recorrência de eclampsia foi definida pela ocorrência de novos episódios convulsivos durante a dose de ataque ou de manutenção do sulfato de magnésio.

De acordo com o protocolo do serviço, as pacientes foram submetidas a medidas para estabilização e suporte que incluem: oxigenação através de cateter nasal, terapia anticonvulsivante com sulfato de magnésio e sondagem vesical de demora. O esquema de Zuspan foi escolhido para a utilização do sulfato de magnésio, com manutenção da medicação $24 \mathrm{~h}$ após o parto. ${ }^{5}$ Recomenda-se suspensão da medicação se ocorre diminuição dos reflexos patelares, diurese $\leq 30 \mathrm{ml} / \mathrm{h}$ e/ou frequência respiratória $\leq 16 \mathrm{ipm}$, pelo risco de parada respiratória pela toxicidade da droga. A resolução da gestação foi indicada depois da estabilização da paciente, sendo a via de parto definida de acordo com o índice de Bishop e do bem-estar fetal.

O protocolo do serviço recomenda que a recorrência da eclampsia deve ser tratada inicialmente com repetição da dose de ataque do sulfato de magnésio e, se houver manutenção do quadro, administração de fenitoína $(750 \mathrm{mg}$ endovenoso nas pacientes com menos de $50 \mathrm{~kg}$, $1 \mathrm{~g}$ nas que têm entre 50 e $70 \mathrm{~kg}$ e $1,5 \mathrm{~g}$ nas com mais de $70 \mathrm{~kg}$ ).

No estudo, as pacientes foram classificadas em relação à presença ou não de recorrência da crise convulsiva. Nessas, foi avaliada a relação da recorrência com a dose do sulfato de magnésio (ataque ou manutenção) e com o momento do parto (anteparto, durante o parto ou pós-parto). Também foi analisada a medicação utilizada no tratamento da recorrência (repetição da dose de ataque do sulfato de $\mathrm{Mg}$, diazepam ou fenitoína).

As características das pacientes avaliadas incluíram: idade materna, idade gestacional, paridade, índice de massa corpórea e pressão arterial durante admissão à maternidade. 
As complicações clínicas estudadas foram: insuficiência renal aguda, edema agudo de pulmão, tromboembolismo pulmonar, síndrome HELLP, hemoperitônio, descolamento de retina, leucoplasia posterior reversível, cardiopatia periparto e descolamento prematuro de placenta.

A pesquisa foi aceita pelo Comitê de Ética e Pesquisa da Maternidade Escola Assis Chateaubriand/Universidade Federal do Ceará. A análise dos dados foi efetuada através do software SPSS (Statistical Package for the Social Sciences) versão 10 e os testes $\mathrm{x}^{2}$ de Fisher foram usados para a comparar as variáveis, a um nível de significância de $5 \%$.

\section{RESULTADOS}

Foram estudadas 69 pacientes com diagnóstico de eclampsia que utilizaram sulfato de magnésio. Destas, seis $(8,7 \%)$ apresentaram recorrência da convulsão, sendo que quatro $(66,7 \%)$ pacientes apresentaram nova crise convulsiva na fase de manutenção do sulfato e duas $(33,3 \%)$ após finalizar a medicação. Em relação ao parto, duas (33,3\%) pacientes apresentaram recorrência antes e durante o parto e quatro $(66,7 \%)$ no puerpério (Tabela 1$)$. O intervalo entre a dose de ataque do sulfato de magnésio e a nova crise convulsiva variou de 2 a 144 horas.
Ao analisar a medicação utilizada no tratamento da recorrência da crise convulsiva, identificou-se que foi administrado diazepam em uma paciente $(16,7 \%)$, fenitoína em quatro $(66,7 \%)$ e repetida metade da dose de ataque do sulfato em uma (16,7\%) (Tabela 2). Após a nova terapia, nenhuma paciente apresentou recorrência das convulsões.

Não houve diferença significativa no perfil das pacientes dos grupos com e sem recorrência de eclampsia (Tabela 3). Os grupos foram estatisticamente semelhantes em relação à idade, primiparidade, idade gestacional em semanas, PAS e PAD na admissão, índice de massa corpórea (IMC) e associação com hipertensão arterial sistêmica.

Em relação às complicações maternas, não houve diferença significativa entre a prevalência nos grupos com e sem recorrência de eclampsia, respectivamente: síndrome HELLP $(33,3 \%$ x 20,6\%, p 0,389), insuficiência renal aguda (0 x $3,2 \%$, p 0,832), insuficiência respiratória $(16,7 \% \times 6,3 \%$, p 0,375), tromboembolismo pulmonar ( $0 \times 1,6 \%$, p 0,913), edema agudo de pulmão $(0 \times 3,2 \%$, p 0,832), hemoperitônio $(0 \times 1,6 \%$, p 0,913), descolamento de retina $(0 \times 1,6 \%, p$ 0,913), leucoplasia posterior reversível $(0 \times 3,2 \%, \mathrm{p} 0,832)$, cardiopatia periparto $(0 \times 3,2 \%$, p 0,832$)$ e descolamento prematuro de placenta $(0 \%$ x 6,3\%, p 0,689) (Tabela 4$)$.

Tabela 1. Recorrência da convulsão em pacientes com eclampsia. MEAC-UFC-2013-2016.

\begin{tabular}{lll}
\hline \multicolumn{1}{c}{ Recorrência da convulsão } & N & $\%$ \\
\hline Em relação ao uso do sulfato de magnésio & & \\
- Na fase de manutenção & 4 & $66,7 \%$ \\
- Após fase de manutenção & 2 & $33,3 \%$ \\
Em relação ao parto & & \\
- Pré-parto ou intraparto & 2 & $33,3 \%$ \\
- Pós-parto & 4 & $66,7 \%$ \\
\hline
\end{tabular}

Tabela 2. Tratamento da recorrência da convulsão na eclampsia. MEAC-UFC-2013-2016.

\begin{tabular}{lcc}
\hline Tratamento da recorrência & N & \% \\
\hline Fenitoina & 4 & $66,7 \%$ \\
Diazepam & 1 & $16,7 \%$ \\
Sulfato de magnésio & 1 & $16,7 \%$ \\
\hline
\end{tabular}

Tabela 3. Perfil das pacientes com eclampsia nos grupos com e sem recorrência de eclampsia. MEAC-UFC-2013-2016.

\begin{tabular}{lccc}
\hline Características & \multicolumn{3}{c}{ Recorrência da crise convulsiva } \\
& Sim & Não & p $^{*}$ \\
\hline Idade (média, em anos) & 23 & 21 & 0,655 \\
Primíparas (\%) & 33,3 & 71,4 & 0,077 \\
Idade gestacional (média, em semanas) & 35,3 & 36,2 & 0,570 \\
PAS na admissão (média, em mmHg) & 137 & 146 & 0,452 \\
PAD na admissão (média, em mmHg) & 82 & 93 & 0,212 \\
IMC (média, em Kg/m²) & 25,7 & 26,4 & 0,652 \\
Hipertensão associada (\%) & 0 & 4,8 & 0,758 \\
\hline
\end{tabular}

*O teste qui-quadrado foi utilizado para comparar porcentagem (\%) e o teste de Fisher para comparar média. 
Tabela 4. Complicações clínicas e obstétricas nas pacientes com eclampsia nos grupos com e sem recorrência de eclampsia. MEAC-UFC-2013-2016.

\begin{tabular}{llll}
\hline \multicolumn{1}{c}{ Complicações } & \multicolumn{1}{c}{ Com recorrência } & Sem recorrência & $\mathbf{p}^{*}$ \\
& \multicolumn{1}{c}{$\mathbf{n}(\mathbf{\%})=\mathbf{6}(\mathbf{8 , 7})$} & $\mathbf{n}(\mathbf{\%})=\mathbf{6 3}(\mathbf{9 1})$ & \\
\hline Síndrome HELLP & $2(33,3)$ & $13(20,6)$ & 0,389 \\
Insuficiência renal aguda & 0 & $2(3,2)$ & 0,832 \\
Insuficiência respiratória & $1(16,7)$ & $4(6,3)$ & 0,375 \\
Tromboembolismo pulmonar & 0 & $1(1,6)$ & 0,913 \\
Edema agudo de pulmão & 0 & $2(3,2)$ & 0,832 \\
Hemoperitônio & 0 & $1(1,6)$ & 0,913 \\
Descolamento de retina & 0 & $1(1,6)$ & 0,913 \\
Leucoplasia posterior reversível & 0 & $2(3,2)$ & 0,832 \\
Cardiopatia periparto & 0 & $2(3,2)$ & 0,832 \\
Descolamento prematuro de placenta & 0 & $4(6,3)$ & 0,689 \\
\hline
\end{tabular}

*O teste qui-quadrado foi utilizado.

\section{DISCUSSÃO}

No estudo atual, a porcentagem de recorrência de convulsão durante ou após o uso do sulfato de magnésio foi de 8,7\%, representando 6 casos dentre as 69 pacientes com diagnóstico de eclampsia admitidas na Maternidade Escola Assis Chateaubriand (UFC) em um período de três anos. No período estudado, foram registrados 14.087 nascidos vivos na MEAC, compreendendo uma prevalência de eclampsia de $4,8 / 1.000$ nascidos vivos. A taxa de recorrência de eclampsia mostrou-se similar às taxas relatadas na literatura mundial, por volta de $9,7 \% .{ }^{6}$ De acordo com a literatura, o sulfato de magnésio tem mostrado superioridade em relação aos outros anticonvulsivantes, tanto na prevenção como no tratamento da eclampsia. $^{10}$

Um estudo realizado no Instituto de Medicina Integral Professor Fernando Figueira - IMIP (2000), com amostra de 120 pacientes com eclampsia, apresentou taxa de recorrência de convulsões de $10 \%$ (12 pacientes) após administração do sulfato de magnésio. O tratamento adotado nos casos de recorrência foi a repetição de metade da dose de ataque da medicação. Destas, quatro pacientes apresentaram novas crises convulsivas, sendo submetidas a tratamento anticonvulsivante com diazepam endovenoso. Após o uso do diazepam, uma paciente ainda evoluiu com nova recorrência, sendo tratada com fenitoína. ${ }^{6}$

$\mathrm{Na}$ nossa pesquisa, apesar de nenhuma das seis pacientes com recorrência ter apresentado nova crise após uso das medicações anticonvulsivantes, foi observada uma deficiência no serviço em seguir o protocolo preconizado. De acordo com nosso protocolo, a recorrência da eclampsia deve ser tratada inicialmente com repetição da dose de ataque do sulfato de magnésio e, se houver manutenção do quadro, recomenda-se a administração de fenitoína.

Estudo realizado pela Cochrane, em 2010, comparou o efeito do sulfato de magnésio com a fenitoína no tratamento de mulheres com eclampsia. Na pesquisa envolvendo seis ensaios, com 972 mulheres, o uso do sulfato de magnésio foi associado com redução importante da recorrência de eclampsia (RR 0,34, IC 95\% - 0,24 a 0,49). ${ }^{11}$

Em outra pesquisa, comparando o sulfato de magnésio com o diazepam, com 1.441 mulheres, o emprego do sulfato de magnésio foi associado com redução da morte materna quando comparado ao diazepam (seis ensaios, 1.336 mulheres; RR 0,44, IC 95\%- 0,37 a 0,94). Houve também redução importante no risco de recorrência de eclampsia (sete ensaios, 1.441 mulheres; RR 0,44, IC 95\% 0,34 a 0,57). ${ }^{12}$

Em 2000, um estudo prospectivo envolvendo pacientes com eclampsia fez uma comparação da eficácia do sulfato de magnésio e da fenitoína no tratamento das convulsões. Das 77 mulheres com eclampsia tratadas com uma das duas medicações, observou-se que, no grupo que utilizou o sulfato de magnésio, $19,5 \%$ das mulheres apresentaram novos episódios convulsivos, já no grupo tratado com fenitoína, $36,1 \%$ manifestaram recorrência. ${ }^{13}$

Estudos mostram que os fatores de risco para eclampsia incluem: pacientes jovens ou com idade avançada (mais em pacientes jovens, quando comparadas com pacientes de idade avançada), nulíparas, obesidade prévia à gestação, ganho de peso excessivo durante a gravidez e hipertensão crônica associada. ${ }^{14}$ No entanto, Yamada, et al (2011), ${ }^{15}$ relataram que aproximadamente $20-50 \%$ das mulheres não apresentaram hipertensão até a última visita pré-natal uma semana antes do seu primeiro episódio convulsivo.

Esakoff, et al. (2015), ${ }^{16}$ mostraram em um estudo retrospectivo de coorte realizado na Califórnia, envolvendo 143.093 mulheres com hipertensão gestacional e pré-eclâmpsia, que a hipertensão arterial sistêmica estava associada com aproximadamente $90 \%$ de diminuição do risco de eclampsia. $\mathrm{O}$ nosso estudo mostrou que apenas 5,8\% das pacientes admitidas com diagnóstico de eclampsia apresentavam hipertensão arterial sistêmica prévia associada. 
As complicações da eclampsia estudadas não apresentaram diferença estatisticamente significante entre os dois grupos pesquisados. Deve-se considerar que o grupo que apresentou recorrência de eclampsia estava representando por uma amostra pequena (seis pacientes), o que pode ser atribuído à menor incidência de recorrência do quadro frente ao diagnóstico precoce e intervenção adequada. Há, no entanto, necessidade de aumentar o número de pacientes estudadas, inclusive prospectivamente, para obtenção de resultados mais consistentes.

\section{REFERÊNCIAS}

1. American College of Obstetricians and Gynecologists. Hipertension in pregnancy. American College of Obstetricians and Gynecologists. 2013;122(5):13-15.

2. Melo BC, Amorim MM, Katz L, Coutinho I, Veríssimo G. Perfil epidemiológico e evolução clínica pós-parto na pré-eclampsia grave. Rev Assoc Med Bras. 2009;55(2);175-80.

3. Peraçoli JC, Parpinelli MA. Síndromes hipertensivas da gestação: identificação de casos graves. Rev Bras Ginecol Obstet. 2005;27(10);627-34.

4. Souza AR, Noronha C Neto, Coutinho IC, Diniz CP, Lima MM. Pré-eclampsia. Femina. 2006;34(7):499-508.

5. Ruano R, Alves EA, Zugaib M. Sulfato de Magnésio (MgSO4) no tratamento e prevenção da eclampsia: qual esquema adotar? Rev Assoc Med Bras. 2004;50(3);229-51.

6. Amorim MM, Santos LC, Porto AM, Martins LK, Vieira V. Recorrência de crise convulsiva após terapia anticonvulsivante com sulfato de magnésio em pacientes com eclampsia. Rev Bras Ginecol Obstet. 2000;22(3);159-65.

7. Souza AS, Amorim MM, Coelho IC, Lima MM, Noronha C Neto, Figueroa JN. Doppler das artérias umbilicais e cerebral média fetal após sulfato de magnésio na pré-eclampsia. Rev Assoc Med Bras. 2008;54(3);232-7.

8. Noronha C Neto, Souza AS, Amorim MM. Tratamento da préeclampsia baseado em evidências. Rev Bras Ginecol Obstet. 2010;32(9);459-68.

\section{CONCLUSÃO}

A recorrência da crise convulsiva mostrou taxas similares às relatadas na literatura, considerando a padronização na administração do sulfato de magnésio em todas as pacientes acometidas com a patologia. Não houve aumento da morbidade materna nas pacientes com recorrência da eclampsia quando comparadas àquelas sem recorrência dos episódios convulsivos.

9. Pereira MN, Montenegro CA, Rezende J Filho. Síndrome HELLP: diagnóstico e conduta. Femina. 2008;36(2);111-16.

10. Gracia PV, Ludmir J. The use of magnesium sulphate for women with severe preeclampsia or eclampsia diagnosed during the postpartum period. J Matern Fetal Med. 2015;28(18);2007-9.

11. Duley L, Henderson-Smart D, Chou D. Magnesium sulphate versus phenytoin for eclampsia. Cochrane Database Syst Rev. 2010;(10):CD000128.

12. Duley L, Henderson-Smart DJ, Walker GJ, Chou D. Magnesium sulphate versus diazepam for eclampsia. Cochrane Database Syst Rev. 2010;(12):CD000127.

13. Valadares JD Neto, Bertini AM, Taborda WC, Parente JV. Tratamento da eclâmpsia: estudo comparativo entre o sulfato de magnésio e a fenitoína. Rev Bras Ginecol Obstet. 2000;22(9):543-9.

14. Coghill AE, Hansen S, Litman A. Risk factors for eclampsia: a population-based study in Washington State, 1987-2007. Am J Obstet Gynecol. 2011;205(6):553.e1-7.

15. Yamada R, Kuwata T, Matsuda H, Deguchi K, Morikawa M, Yamada T, et al. Risk factors of eclampsia other than hypertension: pregnancy-induced antithrombin deficiency and extraordinary weight gain. Hypertens Pregnancy. 2012;31(2):268-77.

16. Esakoff TF, Rad S, Burwick RM, Caughey AB. Predictors of eclampsia in California. J Matern Fetal Med. 2015;27(10):1531-5.

\section{Como citar:}

Silva FM, Carvalho RH Neto, Feitosa FE. Complicações clínicas e obstétricas em pacientes com eclampsia com e sem recorrência de convulsões após tratamento com sulfato de magnésio. Rev Med UFC. 2018 jul-set;58(3):44-48. 